\title{
Forward Forecast of Stock Price Using LSTM Machine Learning Algorithm
}

\author{
Kavitha Esther Rajakumari, M. Srinivasa Kalyan, and M. Vijay Bhaskar
}

\begin{abstract}
Stock market prediction is the demonstration of attempting to decide the future estimation of an organization stock or other monetary instrument exchanged on a trade. This paper will exhibit how to perform stock expectations utilizing Machine Learning calculations. Foreseeing securities exchange costs is an intricate assignment that generally includes broad human-PC communication. Because of the connected idea of stock costs, customary bunch preparing techniques can't be used productively for securities exchange examination. In the current framework, the Sliding window calculation is used. This calculation investigates the information, with a window pushing ahead, in the wake of examining the information. It is very tedious for expectation of stocks. While, in the proposed framework, the utilization of LSTM (Long Short Term Memory) calculation, gives compelling outcomes. While analyzing, the superfluous information is overlooked. The current framework is additionally not viable, in taking care of non-straight information. What's more, it is less proficient contrasted with LSTM algorithm. So, to help defeat these, LSTM helps in dealing with the information in a productive way. Indeed, speculators are exceptionally intrigued by the exploration zone of stock value expectations. For decent and fruitful speculation, numerous financial specialists are sharp in knowing the future circumstance of the share trading system. Great and viable expectation frameworks for securities exchange encourage brokers, financial specialists, and investigators by giving steady data like the future course of the share trading system. In this work, an intermittent neural system (RNN) and Long Short-Term Memory (LSTM) are presented, a way to deal with anticipate securities exchange lists. The proposed model is a promising prescient procedure for a very non-direct time arrangement, whose designs are hard to catch by customary models.
\end{abstract}

Index Terms-Artificial neural network, closing price prediction, Google trends, long short-term memory, stock market analysis.

\section{INTRODUCTION}

Estimating citations of recorded organizations isn't just a fascinating subject for financial specialists yet additionally for researchers. Apparently disordered information contains regularly in actuality profitable data that can be extricated utilizing specific information investigation techniques, for example, specialized and basic examination. In the present plan, speculators get huge measure of data in an alternate configuration. These are information in numerical frame

Manuscript received January 9, 2020; revised March 13, 2020.

Kavitha Esther Rajakumari is with Dept. of CSE, KCG College of Technology, Chennai, India (e-mail: kavitha.cse@kcgcollege.com).

M. Srinivasa Kalyan is with Software Developer, Vidal Health, Bangalore, India (e-mail: m.srinivasakalyan123@gmail.com).

M. Vijay Bhaskar is with Software Developer, NTT DaTa, Bangalore, India (e-mail: malempativ8@gmail.com). (citations, pointers) and content (distributions, remarks, messages distributed by the organization). In such circumstances, the issue of viable handling and choice emerges. An extra issue is likewise the way that it is important to successfully consolidate different data organizations, for example, numbers and content. Thus, present day data frameworks are incredibly useful instrument for speculators, that help their money related choices. Speculators look at monetary resources that they characterize and screen. At that point, they contrast the present cost and the market cost.

At the point when an organization is esteemed beneath the evaluated esteem, the speculator considers this as a purchase flag. In any case, in the contrary circumstance when the valuation of the organization is more than the present esteem, a business flag is produced. Alongside of this speculators decide the quality of organizations, what for they have to determine the accompanying issues: rates, industry development, profit conjectures and money turnover.

Vivek Rajput et al., originates from literary information. Data from quarterly reports or breaking news stories can significantly influence the offer cost of a security. Most existing writing on monetary content mining depends on recognizing a predefined set of catchphrases and machine learning procedures. These strategies normally allocate loads to watchwords in extent to the development of an offer cost. These kinds of investigations have appeared unmistakable, however frail capacity to figure the heading of offer costs. Monetary markets are profoundly unstable and produce tremendous measures of information day by day. Speculation is a responsibility of cash or different assets to get benefits later on. Stock is one kind of securities. It is the most famous monetary market instrument and its esteem changes rapidly. It tends to be characterized as an indication of capital cooperation by an individual or a venture in an organization or a restricted obligation organization.

Money markets gives chances to merchants and organizations to make ventures on impartial ground Stock costs are anticipated to decide the future estimation of organizations' stock or other budgetary instruments that are advertised on money related trades. Be that as it may, the share trading system is described by non linearity, discontinuities, and high-recurrence multi-polynomial segments since it connects with numerous components, for example, political occasions, general financial conditions, and dealers' desires. Consequently, making exact expectations of stock qualities are testing. Financial specialists can purchase stocks that are identified with the development firms that structure framework ventures, 
employ temporary workers and handle administrative work, and leaders of development firms can purchase stocks from different organizations. At the point when the heading of the market is effectively anticipated, speculators might be better guided and money related prizes will be generous. The test in the present condition, where terrible news can generally be heard, is to gauge proactively, instead of responsively. Along these lines, development organizations are attempting to foresee stock costs which is imperative to be considered on a monetary trade, against sudden drops in the market.

Time arrangement determining comprises in an examination territory intended to take care of different issues, mostly in the monetary region. It is significant that this territory regularly utilizes apparatuses that help with arranging and settling on choices to limit speculation dangers. This goal is clear when one needs to break down money related markets and, thus, it is important to guarantee a decent precision in estimating errands. As indicated by Saini (2016), guaging dependent on a period arrangement speaks to a methods for giving data and learning to help an ensuing choice. Consequently, the examination of time arrangement centers around accomplishing reliance connections among chronicled information. The two general classifications of determining models are direct and nonlinear. For a long time, conventional factual guaging models in monetary designing were straight. Some notable factual models can be utilized in time arrangement estimating. Customary displaying strategies, for example, the Box-Jenkins auto regressive coordinated moving normal, are not sufficient for securities exchange value anticipating.

Machine learning (ML) is making its mark that can play a key in a wide scope of basic applications. In machine learning, bolster vector machines (SVMs) have many propelled highlights that are reflected in their great speculation limit and quick calculation. They are additionally not exceptionally touchy to presumptions about mistake terms and they can endure commotion and riotous segments. Remarkably, SVMs are progressively utilized in materials science, the structure of building frameworks and budgetary hazard forecast.

Speculators as a rule are supporters of either specialized or key examination. In this paper another methodology for stock trade information examination is proposed. The created choice calculation depends on information that originates from two unique sources: specialized examination and principal investigation. The displayed arrangement is a continuation of the work that was done before. The principle oddity is utilization of the LSTM (Long Short-Term Memory) recursive neural system at shutting costs expectation. This sort of ANN (Artificial Neural Network) is reasonable to recognize consecutive conditions in time arrangement. Furthermore another choice calculation has been structured, which depends on LSTM gauge and content examination that utilizes Google Trends alongside information distributed by bankier.pl.

\section{RELATED WORK}

Kannan, Sekar, Sathik and P. Arumugam in [1] utilized information mining innovation to find the concealed examples from the memorable information that have plausible prescient ability in their speculation choices. The expectation of securities exchange is testing undertaking of money related time arrangement forecasts. Jing Tao Yao and bite Lim tan in [2] utilized counterfeit neural systems for order, forecast and acknowledgment. Neural system preparing is a workmanship. Exchanging dependent on neural system yields, or exchanging procedure is likewise a workmanship. Writers talk about a seven-advance neural system expectation show building approach in this article. Pre and post information preparing/investigation abilities, information testing, preparing criteria and model proposal will likewise be canvassed in this article.

Tiffany Hui-Kuang and Kun-Huang Huarng in [3] utilized neural system in light of their abilities in dealing with nonlinear relationship and furthermore actualize another fluffy time arrangement model to enhance determining. The fluffy relationship is utilized to conjecture the Taiwan stock file. Jigar Patel [4] centers around the errand of anticipating future estimations of securities exchange file. Two files to be specific CNX Nifty and S\&P Bombay Stock Exchange (BSE) Sensex from Indian securities exchanges are chosen for exploratory assessment in [5]. Investigations depend on 10 years of verifiable information of these two records. The paper proposes two phase combination approach including Support Vector Regression (SVR) in the principal arrange.

Ching-Hseue cheng, Tai-Liang chen, Liang-Ying Wei in [6] this paper proposed a mixture guaging model utilizing multi-specialized pointers to foresee stock value patterns. They utilized RST calculation to separate semantic guidelines and use hereditary calculation to refine the extricated standards to show signs of improvement guaging exactness and stock return.

Fazel Zarandi M.H, Rezaee B, Turksen I.B and Neshat E in [7] utilized a sort 2 fluffy principle based master framework is created for stock value investigation. The purposed sort 2 fluffy model applies the specialized and crucial records as the info factors. The model utilized for stock value expectation of a car manufactory in Asia. The yield enrollment esteems were anticipated onto the information spaces to produce the following participation estimations of information factors and tuned by hereditary calculation.

Ingoo Han [8] proposes hereditary calculations (GAs) way to deal with highlight discretization and the assurance of association loads for fake neural systems (ANNs) to foresee the stock value record. In this investigation, GA is utilized not exclusively to enhance the learning calculation, yet in addition to decrease the multifaceted nature in highlight space. Time arrangement expectation systems have been utilized in some certifiable applications, for example, money related market forecast, electric utility load determining, climate and natural state forecast, and unwavering quality anticipating.

Ravi Shankar et al. [9] gives an overview of time arrangement expectation applications utilizing a novel machine learning approach: bolster vector machines (SVM). T. Jan [10] reviews machine learning methods for securities exchange expectation. He present late improvements in securities exchange expectation models, and examine their focal points and inconveniences. Moreover, we research 
different worldwide occasions and their issues on anticipating securities exchanges.

Y. Kara et al. [11] endeavored to create two effective models and thought about their exhibitions in foreseeing the course of development in the day by day Istanbul Stock Exchange (ISE) National 100 Index. The models depend on two grouping strategies, fake neural systems (ANN) and bolster vector machines (SVM).

Bo Qian et al. [12] examined the consistency of the Dow Jones Industrial Average record to demonstrate that not all periods are similarly arbitrary. He utilized the Hurst type to choose a period with incredible consistency. Some inductive machine- learning classifiers-counterfeit neural system, choice tree, and k-closest neighbor were then prepared with these produced examples. Through proper joint effort of these models, he accomplished forecast precision up to 65 percent.

E. Guresan et al. [13] assesses the adequacy of neural system models which are known to be dynamic and viable in securities exchange expectations. The models investigated are multi-layer perceptron (MLP), dynamic fake neural system (DAN2) and the cross breed neural systems which utilize summed up auto regressive restrictive heteroscedasticity $(\mathrm{GARCH})$ to separate new information factors. The correlation for each model is done in two view focuses: Mean Square Error (MSE) and Mean Absolute Deviate (MAD) utilizing genuine trade every day rate estimations of NASDAQ Stock Exchange list.

B. Nath et al. [14] manages the use of hybridized delicate registering strategies for mechanized securities exchange estimating and incline investigation. We make utilization of a neural system for one day ahead stock estimating and a neuro fluffy framework for breaking down the pattern of the anticipated stock qualities. Cheng-Yi Tesai et al. [15] hybridizes SVR with oneself arranging highlight delineate strategy and a channel based component choice to decrease the expense of preparing time and to enhance expectation correctness. The cross breed framework directs the accompanying procedures: channel based component determination to pick imperative info qualities; SOFM calculation to bunch the preparation tests; and SVR to anticipate money markets value file. The proposed model was shown utilizing a genuine future data-set - Taiwan record prospects (FITX) to anticipate the following day's value file.

\section{EXISTING SYSTEM}

Customary ways to deal with securities exchange examination and stock value forecast incorporate central investigation, which takes a gander at a stock's past execution and the general validity of the organization itself, and measurable examination, which is exclusively worried about calculating and distinguishing designs in stock value variety. The last is generally accomplished with the assistance of Genetic Algorithms (GA) or Artificial Neural Networks (ANN's), however these neglect to catch connection between stock costs as long haul worldly conditions. Furthermore, in the Existing framework, Sliding window calculation is used. Sliding window is the best approach to rebuild a period arrangement data-set as a directed learning. The utilization of earlier time ventures to anticipate whenever step is called sliding window technique. It might be called window strategy. In measurements and time arrangement investigation, this is known as a slack or slack strategy sometimes.

\section{A. Disadvantages of Existing System}

- Sliding Window forecast frameworks tedious are moderate.

- These frameworks are less proficient in foreseeing.

- Sliding window isn't compelling in taking care of non-direct information.

\section{PROPOSED SYSTEM}

LSTM (Long Short Term Memory) algorithm is used to provide efficient stock price prediction. LSTM algorithm is comparatively faster than sliding window algorithm. It provides more efficient prediction and also suites best for non-linear data. These LSTM play a crucial role in machine learning. In the present version, the results of indicators and oscillators are introduced to the input of LSTM artificial neural network. Whereas the fundamental analysis module has been supplemented by an algorithm that checks users' activity via Google Trends API. All activities are sequentially programmed to take place at the right time of the day using the "Cron" program, which is a time-based application for scheduling tasks in Unix operating systems. Fig. 1 represents an overview of the proposed system. The stock price details from the database are fed to LSTM, to train the data and predict the stocks.

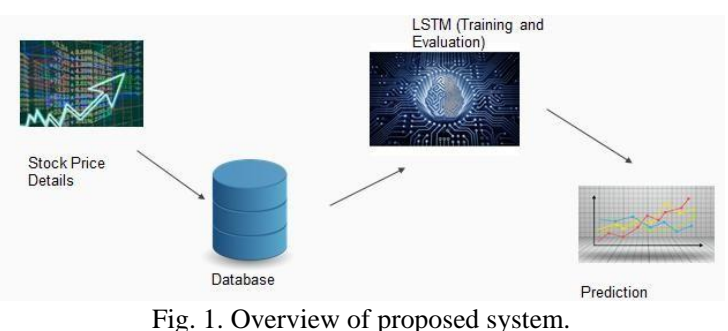

LSTM (Long Short Term Memory) calculation is utilized to give proficient stock value forecast. LSTM calculation is quicker than sliding window calculation. It gives increasingly effective expectation and furthermore suites best for non- straight information. These LSTM assume a pivotal job in machine learning. In the present form, the consequences of pointers and oscillators are acquainted with the contribution of LSTM counterfeit neural system. Though the basic investigation module has been enhanced by a calculation that checks clients' movement through Google Trends API. All exercises are consecutively modified to occur at the correct time utilizing the "Cron" program, which is a time sensitive application for planning errands in Unix working frameworks.

\section{A. Advantages of Proposed System}

- LSTM calculation is comparatively quicker than Sliding window calculation.

- $\quad$ LSTM gives increasingly proficient forecast.

- $\quad$ LSTM suites best for non direct information. 


\section{B. Long Short-Term Memory}

Long Short-Term memory is a standout amongst the best RNNs structures. LSTM presents the memory cell, a unit of calculation that replaces conventional counterfeit neurons in the concealed layer of the system. With these memory cells, systems can successfully relate recollections and information remote in time, consequently suit to get a handle on the structure of information powerfully after some time with high forecast limit.

LSTM's are a unique subset of RNN's that can catch setting explicit transient conditions for extensive stretches of time. Each LSTM neuron is a memory cell that can store other data i.e., it keeps up its own cell state. While neurons in typical RNN's just take in their past concealed state and the present contribution to yield another shrouded express, a LSTM neuron additionally takes in its old cell state and yields its new cell state.

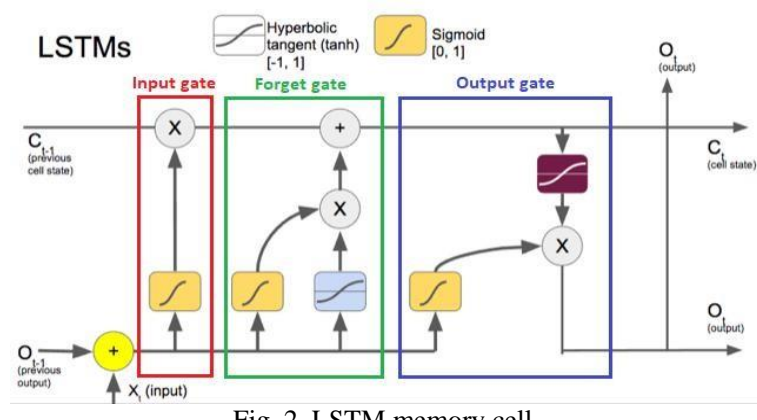

Fig. 2. LSTM memory cell.

A LSTM memory cell, as delineated in Figure 2, has the accompanying three segments

1. Forget gate: the overlook entryway chooses when explicit segments of the cell state are to be supplanted with later data. It yields esteems near 1 for parts of the cell express that ought to be held, and zero for qualities that ought to be disregarded.

2. Input gate: dependent on the info (i.e., past yield o(t-1), input $\mathrm{x}(\mathrm{t})$, and past cell state $\mathrm{c}(\mathrm{t}-1))$, this area of the system learns the conditions under which any data ought to be put away (or refreshed) in the cell state.

3. Output gate: contingent upon the information and cell express, this bit chooses what data is proliferated forward (i.e., yield $\mathrm{o}(\mathrm{t})$ and cell state $\mathrm{c}(\mathrm{t})$ ) to the following hub in the system.

Consequently, LSTM systems are perfect for investigating how variety in one stock's cost can influence the costs of a few different stocks over an extensive stretch of time. They can likewise choose (in a dynamic mold) for to what extent data about explicit past patterns in stock value development should be held so as to all the more precisely anticipate future patterns in the variety of stock costs.

\section{Advantages of LSTM}

The principle favorable position of a LSTM is its capacity to learn setting explicit fleeting reliance. Each LSTM unit recalls data for either a long or a brief time frame (thus the name) without expressly utilizing an enactment work inside the intermittent segments.
An essential certainty to note is that any cell state is duplicated just by the yield of the overlook door, which fluctuates somewhere in the range of 0 and 1 . That is, the overlook door in a LSTM cell is in charge of both the loads and the actuation capacity of the cell state. In this way, data from a past cell state can go through a cell unaltered as opposed to expanding or diminishing exponentially at each time- step or layer, and the loads can join to their ideal qualities in a sensible measure of time. This enables LSTM's to tackle the evaporating slope issue - since the esteem put away in a memory cell isn't iteratively changed, the inclination does not disappear when prepared with back propagation. Furthermore, LSTM's are additionally moderately obtuse to holes (i.e., time slacks between information focuses) contrasted with other RNN's.

\section{SYSTEM DESIGN}

\section{A. Modules Description \\ 1) Download share value information}

Offer cost is the cost of an individual organization. It comprises of date, most elevated cost, least value, close value, number of exchanges, number of offer. Information is separated from Bombay Stock Exchange (BSE). This information helps in preprocessing and preparing the model.

2) Data preprocessing

The way toward changing crude information into reasonable information is called as information preprocessing. Information preprocessing gets ready crude information for further preparing. It is utilized in database driven applications, for example, client relationship the board and principle based applications (neural systems). The information preprocessing is an essential advance before preparing the information. The information preprocessing has the accompanying advances:

\section{- Data cleaning}

It is the way toward investigating, distinguishing and revising chaotic, crude information. Clean information is necessary for good investigation.

\section{- Data integration}

It is the blend of specialized and business forms. This procedure winds up noteworthy in assortment of circumstances which incorporate both business and logical areas.

\section{- Data transformation}

The way toward changing over information from one configuration to other is called information change. It can transform information into auspicious experiences which can affect business decidedly.

- Data reduction

The way toward lessening the measure of limit required to store information. It can expand capacity effectiveness and furthermore diminishes the expense. It can likewise be characterized as changing masses of information into modest number of outlined reports.

\section{3) Train the model}

The way toward preparing includes furnishing a Machine learning calculation with preparing information. The learning calculation discovers designs in the preparation information that delineate info information credits to the 
objective. This progression is exceptionally significant for foreseeing the information.

\section{4) Run LSTM}

LSTM implies Long Short Term Memory. This calculation has three doors in particular Input entryway: Takes information and procedures the information. Yield door: Gives the outcome. Disregard entryway: Forgets the superfluous information. There are associations into and out of the LSTM entryways, a couple of which are repetitive. The loads of these associations, which should be mastered amid preparing, decide how the entryways work. The info door controls the degree to which another esteem streams into the cell, the overlook entryway controls the degree to which an esteem stays in the cell and the yield entryway controls the degree to which the incentive in the cell is utilized.

\section{5) Visualising the predictions}

When the LSTM show is fit to the preparation information, it very well may be utilized to anticipate the finish of-day stock cost of a discretionary stock. This expectation can be performed in two different ways:

- Static - a straightforward, less exact strategy where the model is fit on all the preparation information. Each new time step is then anticipated each one in turn from test information.

- Dynamic - an intricate, increasingly exact methodology where the model is refit for each time venture of the test information as new perceptions are made accessible.

The exactness of the expectation model would then be able to be evaluated vigorously utilizing the RMSE (Root Mean Squared Error) metric. This is because of the way that neural systems when all is said in done (counting LSTM) will in general give diverse outcomes with various beginning conditions on similar information.

At that point rehashing of the model development and forecasting a few times (with various beginning conditions) and after that take the normal RMSE as a sign of how well the arrangement would be relied upon to perform on inconspicuous certifiable stock information (Fig. 3). That is, we will contrast our expectations and real patterns in stock value development that can be deduced from verifiable information.

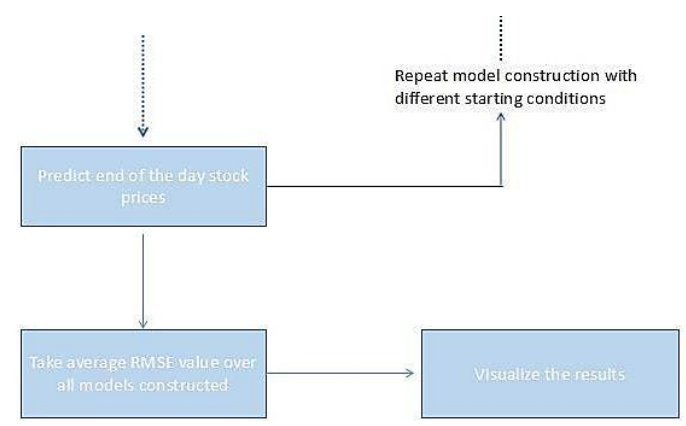

Fig. 3. Prediction of end-of-day stock prices.

When LSTM prediction, is compared withGoogle prediction, the graph is as plotted.

In Fig. 4, the blue line represents the predicted graph, which is quite close to Google's graph.

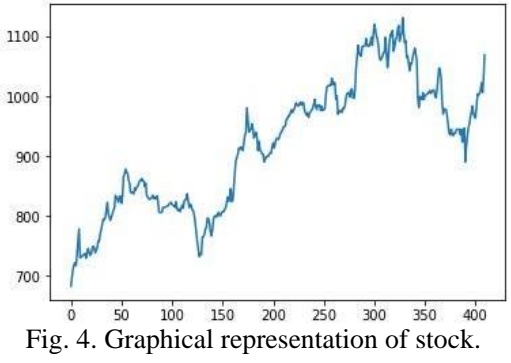

\section{CONCLUSION AND FutURE WORK}

Choice to purchase or move a stock is extremely muddled since numerous components can influence stock cost. This work shows a novel methodology Forward Forecast of Stock Price utilizing LSTM Machine Learning Algorithm. The ubiquity of securities exchange exchanging is developing quickly, which is urging specialists to discover new strategies for the expectation utilizing new systems. The results of comparison between Long Short Term Memory (LSTM) and Sliding window, show that LSTM has a better prediction accuracy .The guaging system isn't just helping the scientists however it likewise helps financial specialists and any individual managing the stock exchange. So as to help foresee the stock records, a guaging model with great precision is required. In this work, we have utilized a standout among the most exact estimating innovation utilizing Recurrent Neural Network and Long Short-Term Memory unit which helps financial specialists, experts or any individual keen on putting resources into the share trading system by giving them a decent learning of things to come circumstance of money markets.

As it's well known, the stock market is a reflection of human emotions, though analysis has it's own limitations. An extension of this stock prediction system would be to feed data from social media for sentimental analysis. It can be linked with the LSTM to train weights and further, improve accuracy.

\section{CONFLICT OF INTEREST}

The authors declare no conflict of interest.

\section{AUTHOR CONTRIBUTIONS}

M. Vijay Bhaskar and M. Srinivasa Kalyan conceived of the presented idea. Dr. Kavitha Esther Rajakumari developed the theory and performed the computations. M. Srinivasa Kalyan and M. Vijay Bhaskar verified the analytical methods. Dr. Kavitha Esther Rajakumari encouraged both other authors to investigate LSTM Machine Learning Algorithm and supervised the findings of this work. All the authors discussed the results and contributed to the manuscript. M. Vijay Bhaskar and M. Srinivasa Kalyan carried out the experiment. Dr. Kavitha Esther Rajakumari wrote the manuscript. M. Vijay Bhaskar developed the theoretical formalism, performed the analytic calculations and M. Srinivasa Kalyan performed the numerical simulations. Dr. Kavitha Esther Rajakumari, M. Vijay Bhaskar., and M. Srinivasa Kalyan. contributed to the design and implementation of the research, to the analysis of 
the results and to the writing of the final manuscript.

\section{REFERENCES}

[1] Kannan, K. Senthamarai, P. S. Sekar, M. M. Sathik, and P. Arumugam, "Financial stock market forecast using data mining techniques," in Proc. the International Multiconference of Engineers and Computer Scientists, 2010.

[2] J. T. Yao and C. L. Tan, "Guidelines for financial prediction with artificial neural networks," in Proc. 8th National Conference on Artificial Intelligence, 2009.

[3] H.-K. T. Yu and K.-H. Huarng, "A neural network-based fuzzy time series model to improve forecasting," Expert Systems with Applications, vol. 37, no. 4, pp. 3366-3372, 2010.

[4] P. Jigar, S. Shah, P. Thakkar, and K. Kotecha, "Predicting stock market index using fusion of machine learning techniques," Expert Systems with Applications, vol. 42, no. 4, pp. 2162-2172, 2015.

[5] S.-G. Deng and Y. Tsung, "Using least squares support vector machines,” J. Chung Cheng Inst. Tec., vol. 40, no. 1, pp. 1-16, 2011.

[6] C.-H. Cheng, T.-L. Chen, and L.-Y. Wei, "A hybrid model based on rough sets theory and genetic algorithms for stock price forecasting," Information Sciences, vol. 180, no. 9, pp. 1610-1629, 2010.

[7] Zarandi, M. H. Fazel, B. Rezaee, I. B. Turksen, and E. Neshat, "A type-2 fuzzy rule-based expert system model for stock price analysis," Expert Systems with Applications, vol. 36, no. 1, pp. 139-154, 2009.

[8] K. Kim and I. Han, "Genetic algorithms approach to feature discretization in artificial neural networks for the prediction of stock price index," Expert systems with Applications, vol. 19, no. 2, pp. 125-132, 2000.

[9] Sapankevych, I. Nicholas, and R. Sankar, "Time series prediction using support vector machines: A Survey," IEEE Computational Intelligence Magazine, vol. 4, no. 2, pp. 24-38, 2009.

[10] P. D. Yoo, M. H. Kim, and T. Jan, "Machine learning techniques and use of event information for stock market prediction," in Proc. nternational Conference on Computational Intelligence for Modelling, Control and Automation and International Conference on Intelligent Agents, Web Technologies and Internet Commerce, 2005.

[11] K. Yakup, M. A. Boyacioglu, and Ö. K. Baykan, "Predicting direction of stock price index movement using artificial neural networks and support vector machines: The sample of the Istanbul stock exchange," Expert Systems with Applications, vol. 38, no. 5, pp. 5311-5319, 2011.

[12] Q. Bo, and K. Rasheed, "Stock market prediction with multiple classifiers," Applied Intelligence, vol. 26, no. 1, pp. 25-33, 2007.
[13] G. Erkam, G. Kayakutlu, and T. U. Daim, "Using artificial neural network models in stock market index prediction," Expert Systems with Applications, vol. 38, no. 8, pp. 10389-10397, 2011.

[14] A. Ajith, B. Nath, and P. K. Mahanti, "Hybrid intelligent systems for stock market analysis," in Proc. International Conference on Computational Science, 2001.

[15] C.-L. Huang and C.-Y. Tsai, "A hybrid SOFM-SVR with a filterbased feature selection for stock market forecasting," Expert Systems with Applications, vol. 36, no. 2, pp. 529-1539, 2009.

Copyright (C) 2020 by the authors. This is an open access article distributed under the Creative Commons Attribution License which permits unrestricted use, distribution, and reproduction in any medium, provided the original work is properly cited (CC BY 4.0).

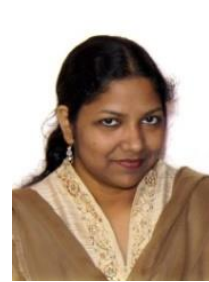

Kavitha Esther Rajakumari has completed her $\mathrm{PhD}$ in the field of software engineering in October 2017. She has published 21 papers in international and national conferences and Scopus indexed journals. Currently her research work is ongoing in the field of software engineering, blockchain, cloud computing and networks. She is a life member in ISTE. She has conducted and organized seminar, guest lecture and certification courses in computer networks, cloud computing and PHP respectively.

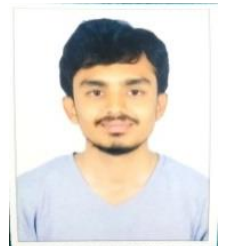

M Srinivasa Kalyan is a software developer currently working at Insurance Tpa Pvt. Ltd. He previously worked in Vidal Health, Bangalore. His work focuses specifically on web development. $\mathrm{He}$ pursued a bachelor's degree from Sathyabama University, Chennai. His research interest topics include machine learning and artificial intelligence.

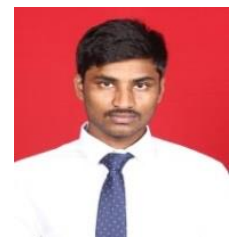

M Vijay Bhaskar is a software developer at NTT Data, Bangalore. He is a self-motivated personality with excellent team work skills. He is currently working on web mining and data analysis. $\mathrm{He}$ pursued a bachelor's degree from Sathyabama University, Chennai. His research interest is in the area of machine learning and software engineering. 